\title{
The pathophysiology of paroxysmal nocturnal hemoglobinuria and treatment with eculizumab
}

This article was published in the following Dove Press journal:

Therapeutics and Clinical Risk Management

18 November 2009

Number of times this article has been viewed

\section{Richard Kelly' \\ Stephen Richards' \\ Peter Hillmen' \\ Anita Hill ${ }^{2}$}

'Institute of Oncology, St. James's University Hospital, Leeds, UK;

${ }^{2}$ Department of Haematology,

Bradford Teaching Hospitals NHS

Foundation Trust, Bradford, UK
Correspondence: Richard Kelly

Department of Haematology, Level

3 Bexley Wing, St. James's University

Hospital, Beckett Street, Leeds LS9 7TF

Tel +44 I I 320685 I3

Fax +44 II 32067468

Email richardkelly@nhs.net

\begin{abstract}
Paroxysmal nocturnal hemoglobinuria is a rare disorder of hemopoietic stem cells. Affected individuals have a triad of clinical associations - intravascular hemolysis, an increased risk of thromboembolism, and bone marrow failure. Most of the symptoms experienced in this disease occur due to the absence of complement regulatory proteins on the surface of the red blood cells. Complement activation is thus not checked and causes destruction of these cells. Eculizumab is a monoclonal antibody treatment which specifically binds to the complement protein $\mathrm{C} 5$, preventing its cleavage, and so halts the complement cascade and prevents the formation of the terminal complement proteins. Eculizumab prevents intravascular hemolysis, stabilizes hemoglobin levels, reduces or stops the need for blood transfusions, and improves fatigue and patient quality of life as well as reducing pulmonary hypertension, decreasing the risk of thrombosis and protecting against worsening renal function. It is not a curative therapy but has a great benefit on those with this rare debilitating condition.
\end{abstract}

Keywords: eculizumab, paroxysmal nocturnal hemoglobinuria, hemolysis

\section{Introduction}

Eculizumab is a humanized monoclonal antibody which binds to the complement protein 5 (C5), thereby inhibiting the formation of the terminal components of the complement cascade. ${ }^{1}$ It was licensed by the Food and Drug Administration in March 2007 and by the European Medicines Agency in June 2007 for the treatment of paroxysmal nocturnal hemoglobinuria (PNH). It has been shown to be a well tolerated and highly effective treatment for patients with $\mathrm{PNH}^{2-4} \mathrm{PNH}$ is a rare hematological disorder where hematological stem cells (HSCs) acquire an abnormality that is then passed on to their progeny. The red blood cells derived from these abnormal HSCs are extremely sensitive to complement mediated lysis which leads to many of the symptoms of the disease. ${ }^{5}$ Prior to eculizumab, treatment was mainly supportive in nature with a median survival of 10 to 15 years for patients treated between 1940 and 1970. ${ }^{6}$ More recently, data from France reported a median survival of 22 years. ${ }^{7}$ This increase in survival may reflect improved supportive care as well as better treatment of more specific complications, such as thromboses.

\section{The genetic defect}

The disease is characterized by hemopoietic clones which harbor somatic mutations of the phosphatidylinositol glycan synthetic pathway due to inactivation of the complementation class A gene $(P I G-A) .{ }^{8}$ The $P I G-A$ gene is one of a number of genes needed for the synthesis of the glycophosphatidylinositol (GPI) anchor within the 
endoplasmic reticulum (ER). ${ }^{9}$ GPI biosynthesis occurs via a stepwise addition of sugar nucleotides and phospholipids within the ER before the completed protein is transferred to the cell surface ${ }^{10}$ (Figure 1). The GPI moiety serves as a membrane anchor for a variety of cell surface proteins. Mutations of the $P I G$ - $A$ gene disrupt the first step of GPI biosynthesis leading to an absence of the GPI anchor and, in turn, a marked deficiency of all GPI linked proteins. ${ }^{11}$

$P I G-A$ is located on the $\mathrm{X}$ chromosome and is monoallelically expressed. All the other genes involved in GPI biosynthesis are autosomal. A single mutation in the $P I G-A$ gene is therefore sufficient to disrupt GPI assembly leading to complete loss of function. For the remainder of genes in this pathway, both alleles would need to be mutated in the same cell to affect GPI production. This explains why all cases of acquired $\mathrm{PNH}$ which have been examined, harbor $P I G-A$ mutations. ${ }^{12,13}$

\section{The complement cascade and hemolysis in PNH}

The complement cascade is an integral part of the innate immune system. It involves sequential reactions that ultimately cause cell lysis either by opsonization and subsequent cell phagocytosis, or by the formation of a phospholipase, called the membrane attack complex (MAC) that punches holes in the cell membrane (Figure 2). CD55 (decay accelerating factor, DAF) and CD59 (membrane inhibitor of reactive lysis, MIRL) are widely expressed on all hemopoietic cells and are both involved in the regulation of complement activation. CD55 increases the removal of $\mathrm{C} 3$ convertase, thereby reducing the amount of $\mathrm{C} 3$ that is cleaved. ${ }^{14}$ CD59 inhibits C9 binding to C5b, C6, C7 and C8

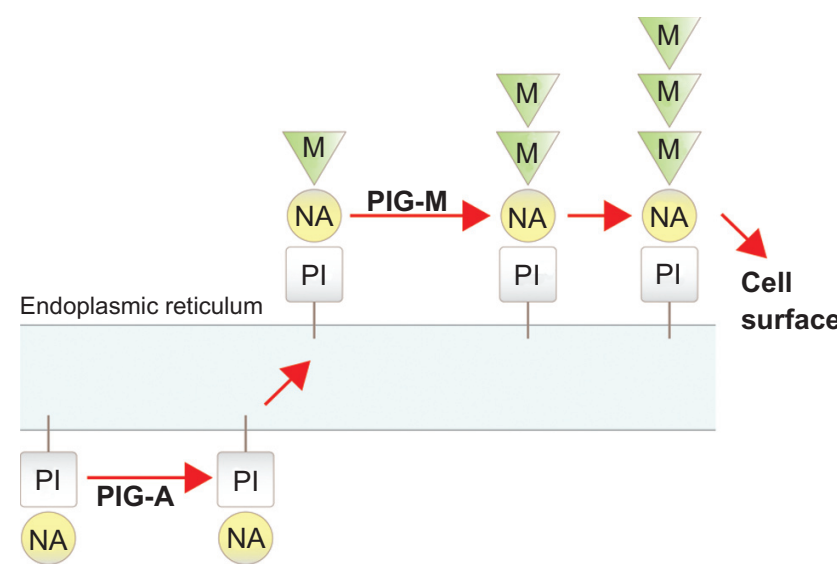

Figure I Glycophosphatidylinositol biosynthesis: an illustration showing the stepwise addition of sugar residues and the sites at which PIG-A and PIG-M are required. Abbreviations: M, mannose; NA, N-acetylglucosamine; PI, phosphatidylinositol. which together make up the MAC. The MAC is then inserted into the cell membrane causing cell lysis.

The absence or reduced expression of CD55 and CD59 on PNH red blood cells leads to their increased sensitivity to complement mediated attack. This in turn causes the majority of symptoms of the disease. Based on their sensitivity to complement attack, erythrocytes in PNH have been classified into 3 groups. ${ }^{14}$ Type I cells are normal red blood cells, type III cells have a complete deficiency of GPI anchored proteins and type II cells have a partial deficiency (Figure 3). The degree of hemolysis suffered by individuals is relative to the proportions of the type II and III cells present. In general, the larger the proportion of type III cells, the more severe the hemolysis suffered by the affected individual.

\section{PNH diagnosis}

As PNH is a rare disease, its precise incidence and prevalence has not been well documented. It is not unusual for patients to be misdiagnosed or remain undiagnosed for long periods. The most reliable data on the incidence and prevalence of the disease is from work undertaken in Yorkshire, England. ${ }^{15}$ In this study, the prevalence of patients with PNH clones of any size is 15.9 per million and the incidence is 1.3 per million of the total population. Eighty-two percent of these patients had a granulocyte clone size greater than $1 \%$, with $43 \%$ of these greater than $10 \%$ and a quarter greater than $50 \%$. Clone sizes down to $0.05 \%$ were detected in this study while evaluating a minimum of $1 \times 10^{6}$ flow cytometry events.

Initially, PNH diagnosis relied on a biochemical assay, the Ham test, in which red blood cells are exposed to acidified serum. Under these conditions, complement is activated via the alternative pathway and causes lysis of PNH cells as they are sensitive to complement attack. ${ }^{16}$ This test was time consuming, non-specific, insensitive and lacked standardization. It was succeeded by flow cytometry in the 1990s.

Flow cytometry is routinely performed to evaluate the size of erythrocyte and granulocyte clones and is considered the "gold standard" for diagnosing PNH. Peripheral blood granulocyte clone size is believed to be the best marker for evaluating the extent of affected HSCs in the bone marrow as the erythrocyte clone size can vary depending both on the degree of intravascular hemolysis present and whether an individual has had a recent red cell transfusion of normal erythrocytes. The granulocyte clone size also correlates well with the platelet clone size.

Initially, flow cytometry was used to look for the absence of specific GPI-linked proteins such as CD55 and CD59 ${ }^{17}$ 


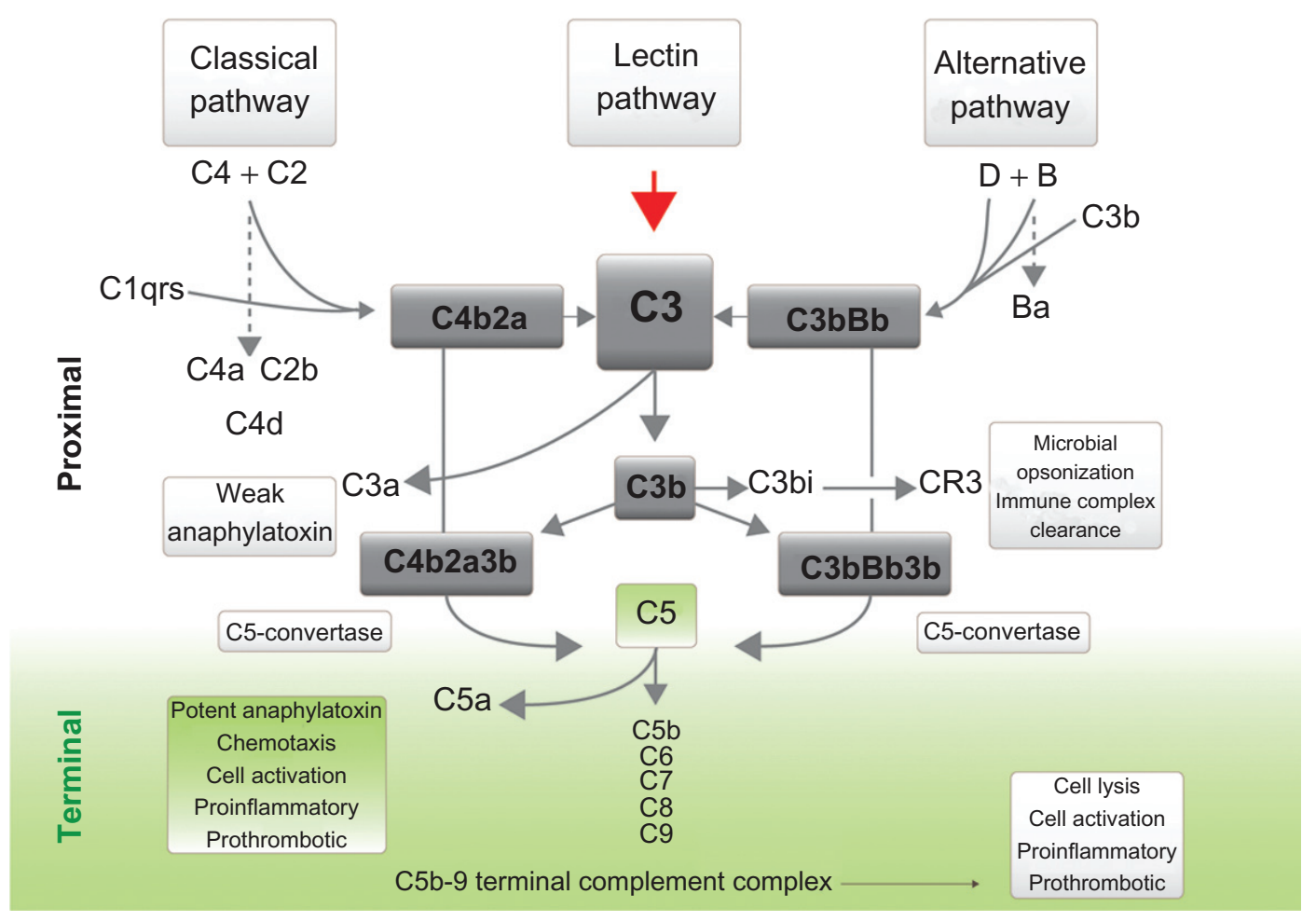

Figure 2 The complement cascade showing the proximal and the terminal complement components which lead to the formation of the membrane attack complex which in turn causes hemolysis of erythrocytes in paroxysmal nocturnal hemoglobinuria.

and determination of the presence of these antigens is still routinely used in evaluating the proportion of $\mathrm{PNH}$ red blood cells present. The use of both of these targets in conjunction excludes rare single antigen deficiencies and allows a red cell clone as small as $0.01 \%$ to be detected. ${ }^{18}$ Evaluation of CD59 expression usually provides the clearest separation of type I, II and III red blood cells (Figure 3).

A wide variety of different GPI-linked proteins have been used to assess the PNH granulocyte clone size. The most recent development in this diagnostic field is the development of the FLAER reagent. ${ }^{18}$ FLAER is a fluorochromelabeled inactive variant of the protein aerolysin which selectively binds directly to the GPI anchor. It cannot be used to ascertain the extent of the PNH red cell clone size as the presence of glycophorin, a non-GPI-linked protein on erythrocytes, binds to the FLAER non-specifically. FLAER can be used alone or in combination with other monoclonal antibodies to GPI-linked antigens to evaluate the granulocyte clone size. At the moment there is no standardization for the diagnosis of PNH with a marked diversity seen in the antibodies used between different centers. FLAER is becoming increasingly used for granulocyte clone evaluation and provides a more accurate assessment, especially of small PNH clones. ${ }^{19}$ In our center, one of the 2 National Centers for treating PNH patients in England, we routinely use 6 color flow cytometry with a combination of FLAER, CD16, CD24, CD33, CD15 and CD14 for granulocyte analysis and CD59, CD55 and CD235a for evaluating erythrocyte clone sizes.

\section{A standardized approach to PNH}

A proposed classification to allow a standard approach to $\mathrm{PNH}$ patients for clinicians is to divide them into three distinct groupings. ${ }^{20}$

1. Classical PNH - these patients have the characteristic symptoms of PNH with intravascular hemolysis and a cellular bone marrow. They have no evidence of any other bone marrow pathology.

2. PNH in the setting of another specified bone marrow disorder - these patients have symptoms of intravascular hemolysis but also have, or have previously had, an underlying bone marrow abnormality such as aplastic anemia (AA) or myelodysplasia (MDS).

3. Subclinical PNH - these patients have no evidence of ongoing hemolysis. They have small PNH clones present and are often seen in patients with bone marrow failure especially in AA and MDS. These patients have been identified due to the development of improved diagnostic flow cytometry as prior to this these small clones 
would have remained undetected. ${ }^{19}$ The significance and relevance of subclinical PNH is unclear and will be evaluated in ongoing studies on otherwise healthy individuals.

The concern with this approach relates to the overlap between those with classical PNH that may have a small degree of aplasia and those with PNH in the setting of another specified bone marrow disorder that may have significant intravascular hemolysis.

\section{The pathogenesis of PNH}

There is good evidence to suggest that all patients who develop PNH have bone marrow failure (usually AA and occasionally MDS) either before or at the time their PNH is diagnosed. ${ }^{21,22}$ Since there is no definitive evidence that GPI deficient cells have an intrinsic survival advantage over normal cells, a cell extrinsic effect is likely to explain the preferential development of PNH clones concurrent with bone marrow failure. ${ }^{22}$ Bone marrow failure, AA in particular, appears to provide the environment needed for the expansion of PNH clones and small PNH clones can be found in up to two thirds of patients with AA. ${ }^{23}$

While the etiology of AA is often poorly defined, a primary role for immune mediated destruction of hemopoietic marrow elements is generally accepted. Consequently one of the main therapies used in AA is immunosuppression, via antithymocyte globulin and ciclosporin. The success of these therapies supports the proposed immune etiology. Furthermore, in vitro experiments demonstrate that removal of T-cells from aplastic bone marrow improves the number of colonies formed in tissue culture and analysis of these

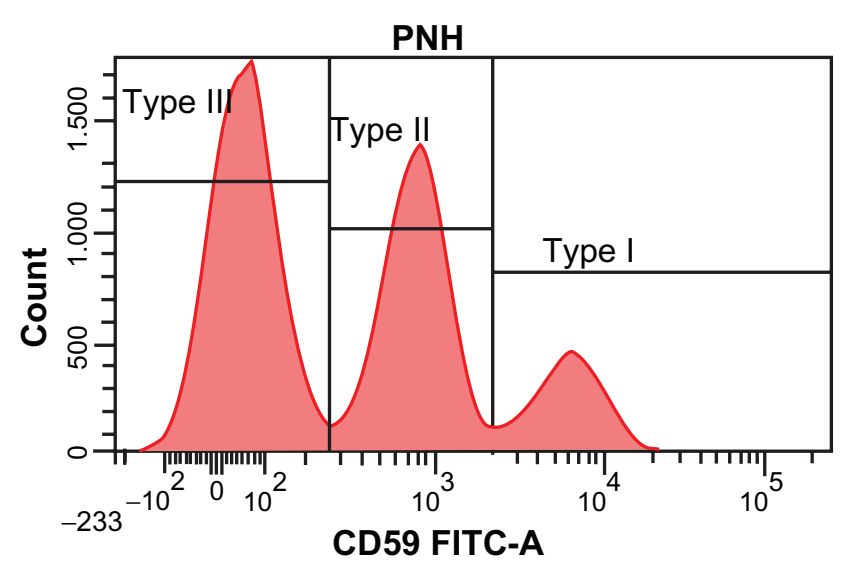

Figure $3 \mathrm{PNH}$ red blood cell analysis showing type III paroxysmal nocturnal hemoglobinuria (PNH) erythrocytes which express no CD59 (shown on the $x$ axis), type II PNH erythrocytes with reduced levels of CD59 and type I erythrocytes with normal levels of CD59.
T-lymphocytes has identified them as activated cytotoxic T-cells (CTL). ${ }^{24}$ The cause of T-cell activation in AA is unknown but drug exposure and viral infection are potential candidates. The role of immune activation in AA leads to the hypothesis that immune evasion is a mechanism for the emergence of the PNH clone.

Some patients with PNH have multiple PNH clones detectable. ${ }^{25-27}$ Multiple clones are also found in those with $\mathrm{PNH}$ who have co-existing AA. ${ }^{28}$ Endo et al reported other features in relation to the presence of multiple clones. ${ }^{26}$ Their study identified four $P I G-A$ mutations in one $\mathrm{PNH}$ patient and they proposed that the phenotypic mosaicism was due to genotypic mosaicism. They hypothesized that this genotypic mosaicism was due to hypermutability of the $P I G-A$ gene.

In cases where multiple clones are present in a patient, one of these often makes up the majority of mature PNH cells. This domination of a single clone may be due to only one of the clones developing in the HSC pool, ${ }^{29}$ whereas the others are generated later during hemopoiesis in progenitor cells. Alternatively, accumulation of a dominant clone may reflect that an additional factor, not related to the $P I G-A$ mutation, is responsible for clonal expansion.

Despite dramatic improvements in treating $\mathrm{PNH}$, the underlying process that determines the expansion of $\mathrm{PNH}$ clones remains unclear. Occurrence of a $P I G-A$ mutant clone is not sufficient for development of PNH, since these occur frequently in the normal population at very low levels. ${ }^{30}$ In view of the association with bone marrow failure, and especially AA, it is likely that expansion of the clone is immune mediated. The simplest theory is that the immune attack in AA requires a GPI-linked protein and that absence of one of these proteins protects PNH cells from attack. Consequently, in the absence of a $P I G$ - $A$ mutant clone, an affected individual would present with AA, but in the presence of a $P I G-A$ mutant clone, hemopoiesis is rescued and the individual presents with $\mathrm{PNH}$, the clinical manifestations of $P I G-A$ deficient hemopoietic cells. ${ }^{25}$ An alternative theory to this, involving the absence of specific GPI-linked proteins which provide a clonal advantage to PNH cells, has also been proposed..$^{31}$ Hanaoka et al proposed that the absence of stress inducible proteins on PNH blood cells provide the basis for clonal expansion. Such a model however does not readily explain the link with $\mathrm{AA}$, the failure of $\mathrm{PNH}$ clones to become dominant in normal individuals, and the lack of intrinsic proliferative advantage in vitro.

Inoue et al proposed a two stage model for the disease whereby clonal selection and clonal expansion occur by two separate events. ${ }^{32}$ In this model, clonal selection relates to 
the $P I G$ - $A$ mutation, occurring in the setting of bone marrow failure with clonal expansion being due to a separate mutation within the $P I G$ - $A$ mutated HSC which confers a proliferative advantage.

Another potential mechanism that has been proposed is that lack of GPI molecules on PNH cells can render them resistant to apoptosis. ${ }^{33,34}$ However, other investigators have failed to observe this difference in apoptosis rates between GPI deficient and normal granulocytes. ${ }^{35}$

Furthermore, mouse models of $P I G-A$ deficiencies do not develop clonal expansion of PNH-like cells. ${ }^{36,37}$ These results argue against a cell intrinsic role for $P I G-A$ deficiency in the development of PNH. One clear explanation for the failure to develop a PNH-like syndrome is that these mouse models do not have underlying bone marrow failure or immune insult. Hence, the conditions for clonal expansion of PNH-like cells are not met.

\section{Clinical symptoms and the role of nitric oxide}

In general, the size of the PNH clone correlates with the degree of symptoms observed. Therefore, patients who have symptomatic PNH tend to have larger clones of PNH cells present. Affected individuals have chronic low grade hemolysis with episodes, or "paroxysms", of severe intravascular hemolysis. During periods of intravascular hemolysis, free hemoglobin is released into the circulation. Haptoglobin, a protein produced mainly by hepatocytes in the liver, rapidly binds free hemoglobin and this haptoglobinhemoglobin complex is then degraded in the liver. This process is overwhelmed in $\mathrm{PNH}$ and leads to excess free hemoglobin avidly and irreversibly binding to nitric oxide (NO) ${ }^{38,39} \mathrm{NO}$ plays an important role in the maintenance of vascular tone by relaxation of smooth muscle which consequently causes blood vessel vasodilation. Depletion of NO in individuals with PNH leads to smooth muscle dystonia and this may be responsible for many of the symptoms of the disease. These include esophageal spasm and dysphagia, abdominal pain, severe lethargy and erectile dysfunction in men. NO depletion is associated with the development of a number of cardiovascular morbidities including pulmonary hypertension. Recently it has been shown that PNH patients have a high prevalence of pulmonary hypertension which is likely secondary to NO depletion. ${ }^{39}$

The clinical outcome of patients with $\mathrm{PNH}$ is highly variable from one individual to the next. Thrombosis remains the commonest cause of death in the disease, occurring in $40 \%$ of patients, with a third of these being fatal. ${ }^{6,7}$ These thromboses predominantly occur in the venous system with an increase in thromboses in typical sites such as deep vein thromboses and pulmonary emboli as well as at unusual sites, such as the hepatic, mesenteric and cerebral veins. ${ }^{6}$ The arterial thrombosis risk is also elevated with increased occurrences of myocardial infarctions and strokes. Hall et al reported the risk of thrombosis in patients with a $50 \%$ or greater $\mathrm{PNH}$ clone to be $44 \%$, and those with a less than 50\% PNH clone to be $5.8 \%{ }^{40}$ Although the risk is far greater in those with larger PNH clones, even those with small clones (as low as $10 \%$ ) have a much higher thrombotic risk when compared with the general population. ${ }^{41,42}$

The underlying mechanisms causing thromboembolism in individuals with $\mathrm{PNH}$ has not been clearly defined and may be multifactorial in nature. NO depletion due to binding of NO to free hemoglobin causes both increased platelet aggregation and adhesion. ${ }^{38}$ Additionally, platelets lacking GPI-linked proteins are susceptible to complement mediated attack which leads to platelet activation and the formation and exocytosis of prothrombotic microvesicles containing the MAC. ${ }^{43}$ These microvesicles have been shown to be present at high levels in the blood of patients with PNH. ${ }^{44}$ Another potential cause for the increased thrombotic risk is due to GPI deficient neutrophils lacking urokinase type plasminogen activator thereby reducing plasminogen activation and causing a reduction in fibrinolysis. ${ }^{45}$ It is likely that the increased thrombotic risk in PNH is due to a combination of these factors rather than a single underlying mechanism.

\section{PNH treatment}

Allogeneic bone marrow transplantation is the only curative therapy for $\mathrm{PNH}^{46-48}$ However it carries a high rate of mortality and morbidity due to infection, graft versus host disease and graft failure. It is only an option for a minority of patients either because they are not suitable candidates for the procedure, a donor is unavailable or treatment with eculizumab may be deemed more appropriate. The introduction of eculizumab treatment plus the fact that around 15\% of patients with $\mathrm{PNH}$ undergo a spontaneous remission of the disease, result in transplantation only being undertaken in specific circumstances. ${ }^{6}$ Transplantation should be considered when there is an associated severe bone marrow failure, life-threatening hemolysis with no access to eculizumab, recurrent thromboses despite eculizumab treatment and in cases of syngeneic twins.

Prior to eculizumab, the mainstay of treatment for patients with PNH has been supportive in nature. Folic acid is routinely taken, as in other hemolytic anemias, in view of the 
increased red cell production. Many patients have become transfusion dependent in an effort to alleviate symptoms related to their anemia. Some of these patients have developed iron overload due to the number of transfusions and require treatment with iron chelation. The majority, however, remain in an iron deficient state due to their persistent hemoglobinuria and therefore need to take oral iron supplements. In those patients with a large PNH clone, warfarin therapy has been employed, which can reduce the risk of developing thrombosis. ${ }^{40}$

\section{Eculizumab}

Eculizumab is a humanized monoclonal antibody that binds to the complement protein $\mathrm{C} 5$ and prevents its cleavage into $\mathrm{C} 5 \mathrm{a}$ and $\mathrm{C} 5 \mathrm{~b}$. It is comprised of murine complementaritydetermining regions within a human antibody framework that includes IgG2 and IgG4 regions. Treatment with eculizumab therefore prevents $\mathrm{C} 5 \mathrm{~b}$ formation which is necessary to form MAC through binding to the complement proteins $\mathrm{C} 6, \mathrm{C} 7$, $\mathrm{C} 8$ and $\mathrm{C}$. As the clinical features in $\mathrm{PNH}$ are caused by the MAC attack on erythrocytes, preventing its formation was likely to protect PNH red blood cells in the circulation (Figure 4). Information on the possible effects eculizumab might have in treating PNH was first seen in a case reported by Yonemura et al of a patient with co-existing PNH and a deficiency of C9. ${ }^{49}$ This patient was well with only mild hemolysis and only became unwell after receiving a whole blood transfusion after an operation. Transfusion of whole blood includes exogenous C9 allowing the symptoms of PNH to manifest.

The importance of both the proximal and the terminal complement proteins can be shown by examining people with rare inherited complement protein deficiencies. Congenital deficiencies of proximal complement proteins result in recurrent severe infections and death early in life. ${ }^{50}$ Deficiencies of the terminal complement proteins, $\mathrm{C} 5, \mathrm{C} 6, \mathrm{C} 7, \mathrm{C} 8$ or C9 increase the susceptibility to infection with the bacteria

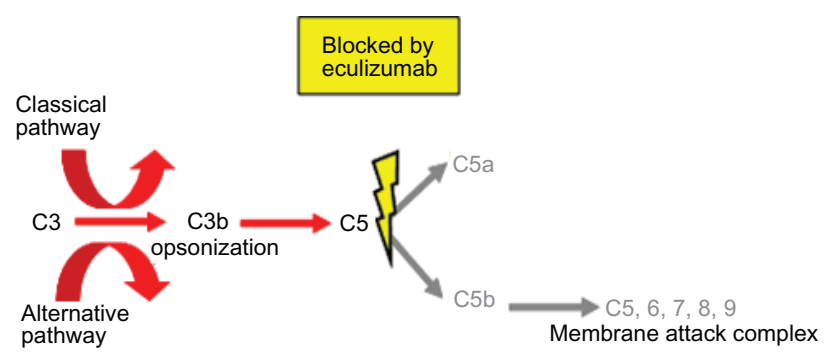

Figure 4 Eculizumab binding to C5 inhibiting formation of the membrane attack complex and release of $\mathrm{C} 5 \mathrm{a}$.
Neisseria meningitidis. ${ }^{50}$ In these individuals however, the proximal complement cascade remains intact allowing the formation of $\mathrm{C} 3 \mathrm{~b}$ and its subsequent opsonization and clearance of most other bacteria.

C5 is a good therapeutic target as all the proximal complement pathways converge at C5 (Figure 2). Complement blockade at C5 will therefore halt the complement cascade preventing activation of the terminal complement components no matter which initial pathway has been activated.

Eculizumab was initially evaluated in the treatment of patients with rheumatoid arthritis and systemic lupus erythematosis. ${ }^{51,52}$ These early clinical studies provided information on the frequency of dosing as well as the doses required to provide complement blockade.

\section{Clinical trials of eculizumab}

\section{The pilot study}

Eleven patients were entered into this initial study in 2002 to assess the effect of eculizumab in $\mathrm{PNH}$ patients. ${ }^{2}$ Individuals needed to have received 4 or greater red blood cell transfusions in the preceding 12 months to enter the study. Patients were also vaccinated against $N$. meningitidis (serotypes A + C).

Eculizumab was administered at a dose of $600 \mathrm{mg}$ weekly for the first 4 weeks followed by a dose of $900 \mathrm{mg}$ every fortnight from the 5 th week onwards. Data were collected on the drug pharmacokinetics and pharmacodynamics, markers of hemolysis (lactate dehydrogenase (LDH), haptoglobin and bilirubin), hemoglobin and reticulocyte levels, PNH clone size proportions as well as the degree of hemoglobinuria and the individual transfusion requirements. European Organization for Research and Treatment of Cancer (EORTC) questionnaires were employed to assess potential changes in quality of life. Concomitant medication such as immunosuppressive and anticoagulant medication was continued.

There was a dramatic reduction in LDH levels in these patients from a mean level of $3111 \mathrm{IU} / \mathrm{L}$ prior to treatment down to $594 \mathrm{IU} / \mathrm{L}$ in the study. The proportion of type III erythrocytes during the 12 weeks increased significantly from a mean of $36.7 \%$ to $59.2 \%$ which supports an increased survival of these cells due to the prevention of intravascular hemolysis. Transfusion requirements reduced in all 11 patients with 5 becoming transfusion independent for the entire trial period. Hemoglobinuria resolved in most and there was a rapid and sustained improvement in quality of life.

On completion of the pilot study these 11 patients all enrolled into a 12 month extension trial to evaluate the 
long-term safety and efficacy of eculizumab. ${ }^{53}$ Complement blockade with a consequent sustained reduction in both intravascular hemolysis and transfusion requirement was observed during the extension period. The initial dramatic improvement in quality of life was also maintained. The use of eculizumab appeared to be safe and well tolerated in these patients.

The pilot study supported the importance of terminal complement activation in PNH and suggested that eculizumab could be used to reduce hemolysis and transfusion requirements whilst increasing the proportion of type III erythrocytes and improving quality of life in this debilitating disease.

\section{The TRIUMPH study}

Following the success of the pilot study, a double-blind, randomized, placebo-controlled multicenter phase 3 trial (TRIUMPH) was undertaken. ${ }^{3}$ Patients could enter into a 3 month observation period if they were more than 18 years old, had received four or more red cell transfusions in the preceding twelve months, had $>10 \%$ PNH type III erythrocytes present, a platelet count $>100 \times 10^{9} / \mathrm{L}$ and an $\mathrm{LDH}$ level 1.5 times the upper limit of normal. Patients that were transfused during the observation period were then eligible to enter the study. Eighty-seven patients were randomized to receive either eculizumab or placebo for 6 months with 44 of these receiving eculizumab and the rest being given placebo infusions. At the end of the 26 week period patients who had been given placebo were allowed to cross-over and receive eculizumab. The protocol for administration of eculizumab was the same as in the pilot trial.

The TRIUMPH study was designed to see if eculizumab stabilized hemoglobin levels and reduced transfusion requirements. Secondary trial endpoints included assessment of intravascular hemolysis, fatigue and quality of life.

Stabilization of hemoglobin levels was achieved in $49 \%$ of the patients treated with eculizumab and this did not occur in any patient in the placebo group. Fifty-one percent of the study group remained transfusion independent throughout the 26-week study whereas all the placebo group patients had been transfused by the 14th week of the study. There was a $44 \%$ reduction in the number of transfusions needed by the patients who did not become transfusion independent on eculizumab. As in the pilot study, there was a marked reduction in intravascular hemolysis in the eculizumab group with LDH levels falling from a mean of 2199.7 IU/L to $327 \mathrm{IU} / \mathrm{L}$. Levels in the placebo group remained high with a mean of 2418.9 IU/L during the 26 weeks. Fatigue was assessed using the Functional Assessment of Chronic Illness Therapy (FACIT) instrument and quality of life by EORTC questionnaires. Both showed huge improvements in those treated with eculizumab. Although the TRIUMPH study was not designed to look at thrombotic events, no thromboses occurred in the patients receiving eculizumab.

This study confirmed the importance of eculizumab in stabilizing hemoglobin levels, reducing or stopping the need for transfusions as well as improving anemia, fatigue and quality of life for PNH patients.

\section{The SHEPHERD study}

The SHEPHERD study was a phase 3 trial designed to evaluate the safety and efficacy of eculizumab in treating a more diverse population of patients with $\mathrm{PNH}$ including those with fewer transfusion requirements and thrombocytopenia. ${ }^{4}$ The trial entry criteria included patients transfused at least once in the preceding 2 years, a platelet count of $>30 \times 10^{9} / \mathrm{L},>10 \% \mathrm{PNH}$ type III erythrocytes present and an LDH level 1.5 times the upper limit of normal. Ninety-seven patients were enrolled and 96 of these completed the 52 week study period. The protocol for administration of eculizumab was the same as in the pilot and the TRIUMPH trials.

Eculizumab was well tolerated with a similar rate of adverse events (AE) reported to the TRIUMPH study. The commonest AEs reported were headaches (53\%), nasopharyngitis (32\%) and upper respiratory tract infections $(30 \%)$. The incidence of headaches was greater in the first 26 weeks (49\%) of the study compared to the last 26 weeks $(15 \%)$. The majority of these headaches were mild to moderate in severity and most occurred only within the first 2 weeks of their eculizumab infusions. Forty-four serious AEs were reported but none were thought to be probably or definitely due to eculizumab though 7 of them were possibly related to the drug. These 7 events included 2 of pyrexia, 1 of headache, 1 of abdominal distension, 1 of a viral infection, 1 of anxiety and 1 of renal impairment. Two patients, both with a prior history of thromboembolism, had a further thrombosis during the study. Eighty-nine patients (91\%) had mild or moderate infections during the study with the majority of these thought to be unrelated to eculizumab. Low titer anti-eculizumab antibodies occurred in 2 patients $(2 \%)$ but with no loss of drug efficacy.

Eighty-nine patients (91\%) had serum eculizumab levels $>35 \mu \mathrm{g} / \mathrm{mL}$, throughout the trial period. This level has been shown to completely block terminal complement activation and prevent intravascular hemolysis. ${ }^{2}$ The other 
8 patients experienced breakthrough hemolysis 1 to 2 days prior to their next eculizumab infusions, but after reducing the dosing interval from 14 to 12 days, consistent complement blockade was resumed. There was a marked reduction in intravascular hemolysis in the eculizumab group with LDH levels falling from a mean of $2201 \mathrm{IU} / \mathrm{L}$ to $297 \mathrm{IU} / \mathrm{L}$ and the proportion of type III erythrocytes present increased from a mean of $33.5 \%$ to $55.7 \%$. Transfusion requirements reduced from a mean of 12.3 units per patient in the 12 months prior to the study to 5.9 units per patient, with $51 \%$ of patients remaining transfusion independent for the entire 52 weeks.

This trial shows that eculizumab appears to be safe and well tolerated and provides marked benefits to a broader group of PNH patients by reducing intravascular hemolysis.

\section{Eculizumab: its effect on thromboembolism and renal function}

Clinical trial patients from all three of these trials were entered into an extension study and the rate of thromboembolism assessed. ${ }^{54}$ Thrombosis rates in these 195 trial patients were compared before and after treatment with eculizumab. Prior to eculizumab treatment this group had a thromboembolic rate of 7.37 thromboses per 100 patient years. This decreased dramatically to 1.07 thromboses per 100 patient years with complement blockade. The question arises as to whether patients that are treated with eculizumab should also receive warfarin. Warfarin has been shown to reduce the risk of thrombosis in PNH but it also carries a significant risk ( $2 \%$ to $13 \%$ ) of severe bleeding. ${ }^{55}$ Patients who have had a prior thrombosis have a high risk of subsequent thrombosis ${ }^{7,56}$ and in view of this, these patients are often treated with both eculizumab and anticoagulation. However it remains unclear whether it is better to use eculizumab alone or in conjunction with anticoagulation in patients with PNH who have not suffered a thrombosis and are deemed to be high-risk.

Renal damage is a common finding in patients with $\mathrm{PNH}$ and this damage may be due to repeated microvascular thrombosis, renal cortical hemosiderosis, repeated exposure of the kidney to heme proteins or reduced kidney perfusion due to the reduced availability of NO. ${ }^{57-60}$ The 195 patients from the PNH clinical trials were screened for evidence of chronic kidney disease (CKD) before and after 18 months of treatment with eculizumab. ${ }^{61}$ Sixty five percent (126/195) of patients had evidence of CKD and administration of eculizumab was generally associated with an improvement in renal function with $21 \%$ of patients with prior CKD no longer classified with CKD during eculizumab treatment. The patients with more mild baseline kidney disease were the most likely to have an improvement in their renal function.

\section{Specific issues on eculizumab treatment}

\section{Neisseria meningitidis infection}

One of the main concerns for physicians treating patients with eculizumab is that of infection with $N$. meningitidis. In the PNH clinical studies, 2 out of 195 patients experienced meningococcal sepsis. Both patients were treated promptly and remain well. Since the completion of these clinical trials there has been 1 fatality due to meningococcal sepsis. The risk of this infection is low and has been further reduced by vaccinating patients with the quadravalent vaccine against serotypes A, C, W and Y, prior to starting eculizumab. Unfortunately at present there is no vaccine against serotype $\mathrm{B}$ which is a common strain of the bacteria in Europe. As in the TRIUMPH and SHEPHERD studies in our center we routinely give the patients two 750-mg doses of ciprofloxacin to keep at home and take if unwell while also seeking medical attention. It is important that both patients and physicians are educated and remain vigilant about the signs of the disease so that they seek and receive prompt targeted medical treatment when needed.

\section{Breakthrough hemolysis}

A significant minority of patients treated with eculizumab develop symptoms of intravascular hemolysis 1 to 2 days prior to their next eculizumab infusion (around 7\%-9\% - personal experience). In some patients this can be resolved by reducing the treatment from every 14 days to every 12 days. ${ }^{2,4}$ Treatment every 12 days can be inconvenient as the day of treatment varies and infusions are also required at weekends. A 1200-mg dosing schedule every 14 days has been used in our center and found to consistently block complement even in patients who still have breakthrough symptoms on 900mg eculizumab every 12 days. ${ }^{62}$ Patients treated with this $1200 \mathrm{mg}$ dose were found to have a good correlation between eculizumab and LDH levels, suggesting that a breakthrough in complement activity due to insufficient drug levels can be monitored by measuring LDH near the end of the dosing interval.

\section{Extravascular hemolysis}

Despite the enormous health improvements seen in most patients with PNH there are some patients that remain anemic and dependent on transfusions. This suboptimal response may be due to the complement protein $\mathrm{C} 3$ binding to $\mathrm{PNH}$ red 
blood cells causing an increase in extravascular hemolysis. ${ }^{63}$ Co-existent bone marrow failure is also likely to be contributory. Eculizumab blocks the formation of the terminal components of the complement cascade but has no effect on the proximal components. The absence of CD55 (DAF) on the surface of the $\mathrm{PNH}$ red cells means the usual regulation and accelerated decay of cleaved $\mathrm{C} 3$ products is disrupted and the red cells are bound with $\mathrm{C} 3$. This phenomenon has emerged with the use of eculizumab as prior to it these blood cells were likely to hemolyzed intravascularly.

\section{Pregnancy}

Pregnancy carries an increased risk of mortality and morbidity in PNH compared with the non-pregnant state. ${ }^{64-66}$ The main risks to the mother are in the form of thromboembolism and infection, whereas in the baby most complications relate to prematurity which occurs in around half of pregnancies to mothers with PNH. Eculizumab has been used in pregnancy in one patient during the third trimester. ${ }^{67}$ It has also been used for the first time from conception and through the entire pregnancy. ${ }^{68}$ In this case eculizumab was not detected in the cord blood or breast milk, suggesting it does not cross the placenta or into mother's milk. The use of eculizumab in pregnancy will become an increasingly frequent conundrum as more young women treated with the drug feel well enough to consider starting a family.

\section{Conclusion and summary}

Eculizumab has dramatically changed the way clinicians approach treatment for patients with PNH. It took just 5 years from the pilot study in 2002 to the drug gaining its Food and Drugs Administration license and its European Medicines Agency license in March and June 2007 respectively. The eculizumab clinical trials have shown that it is safe and well tolerated and provides huge benefits for $\mathrm{PNH}$ patients who previously received supportive therapies. It has been shown to stop intravascular hemolysis and the subsequent symptoms patients develop, reduce or abolish the need for transfusions, stabilize hemoglobin levels, improve patient quality of life, reduce fatigue, reduce the risk of developing thromboses, protect against worsening renal function and decrease pulmonary hypertension. It is likely that in the future eculizumab will improve patient mortality.

Although it has many positive points, there are some negatives to eculizumab treatment. It has to be given as an intravenous infusion every 2 weeks, there is a small but definite increase in susceptibility to $N$. meningitidis, it costs $\sim$ US $\$ 400,000$ per year and it does not cure the disease.
Eculizumab is of use in treating classical PNH, ie, where hemolysis is the predominant disease component. It does not have a role in the treatment of patients with subclinical PNH with no evidence of hemolysis.

Further research into PNH is needed to ascertain why and how it occurs and may allow future therapies to be developed to cure the disease. For the time being, eculizumab provides patients who have previously suffered with a chronic illness the ability to lead normal family and working lives within their communities.

\section{Disclosures}

Richard Kelly, Stephen Richards and Anita Hill have received honoraria for lecture fees and have sat on advisory boards for Alexion Pharmaceuticals. Peter Hillmen has received research funding, honoraria for lecture fees and has sat on advisory boards for Alexion Pharmaceuticals.

\section{References}

1. Thomas TC, Rollins SA, Rother RP, et al. Inhibition of complement activity by humanized anti-C5 antibody and single-chain Fv. Mol Immunol. 1996;33:1389-1401.

2. Hillmen P, Hall C, Marsh JCW, et al. Effect of eculizumab on hemolysis and transfusion requirements in patients with paroxysmal nocturnal hemoglobinuria. $N$ Engl J Med. 2004;350:552-559.

3. Hillmen P, Young N, Schubert J, et al. The complement inhibitor eculizumab in paroxysmal nocturnal hemoglobinuria. $N$ Engl $J$ Med. 2006;355:1233-1243.

4. Brodsky RA, Young NS, Antonioli E, et al. Multicenter phase 3 study of the complement inhibitor eculizumab for the treatment of patients with paroxysmal nocturnal hemoglobinuria. Blood. 2008;111: $1840-1847$.

5. Rosse WF, Dacie JV. Immune lysis of normal human and paroxysmal nocturnal hemoglobinuria (PNH) red blood cells. The sensitivity of PNH red cells to lysis by complement and specific antibody. $J$ Clin Invest. 1966;45:736-748.

6. Hillmen P, Lewis SM, Bessler M, Luzzatto L, Dacie JV. Natural history of paroxysmal nocturnal hemoglobinuria. $N$ Engl $J$ Med. 1995;333:1253-1258

7. de Latour RP, Mary JY, Salanoubat C, et al. Paroxysmal nocturnal hemoglobinuria: natural history of disease subcategories. Blood. 2008;112:3099-3106.

8. Takeda J, Miyata T, Kasagoe K, et al. Deficiency of the GPI anchor caused by a somatic mutation of the PIG-A gene in paroxysmal nocturnal hemoglobinuria. Cell. 1993;73:703-711.

9. Miyata T, Takeda J, Iida Y, et al. The cloning of PIG-A, a component in the early step of GPI-anchor biosynthesis. Science. 1993;259: $1318-1320$

10. Vidugiriene J, Menon AK. The GPI anchor of cell-surface proteins is synthesized on the cytoplasmic face of the endoplasmic reticulum. J Cell Biol. 1994;127:333-341.

11. Kinoshita T, Ohishi K, Takeda J. GPI-anchor synthesis in mammalian cells: genes, their products, and a deficiency. J Biochem. 1997;122: 251-257.

12. Tomita M. Biochemical background of paroxysmal nocturnal hemoglobinuria. Biochim Biophys Acta. 1999;1455:269-286.

13. Rosti V. The molecular basis of paroxysmal nocturnal hemoglobinuria Haematologica. 2000;85:82-87.

14. Rosse WF. Variations in the red cells in paroxysmal nocturnal haemoglobinuria. Br J Haematol. 1973;24:327-342. 
15. Hill A, Platts PJ, Smith A, et al. The incidence and prevalence of paroxysmal nocturnal haemoglobinuria $(\mathrm{PNH})$ and survival of patients in Yorkshire. Blood. 2006;108:985.

16. Rosse WF. Dr Ham's test revisited. Blood. 1991;78:547-550.

17. Hall SE, Rosse WF. The use of monoclonal antibodies and flow cytometry in the diagnosis of paroxysmal noctural hemoglobinuria. Blood. 1996;87:5332-5340.

18. Brodsky RA, Mukhina GL, Nelson KL, Chiurazzi PL, Buckley JT, Borowitz MJ. Improved detection and characterization of paroxysmal nocturnal hemoglobinuria using fluorescent aerolysin. Am J Clin Pathol. 2000;114:459-466.

19. Richards SJ, Hill A, Hillmen P. Recent advances in the diagnosis, monitoring, and management of patients with paroxysmal nocturnal hemoglobinuria. Cytometry B Clin Cytom. 2007;72:291-298.

20. Parker C, Omine M, Richards S, et al. Diagnosis and management of paroxysmal nocturnal hemoglobinuria. Blood. 2005;106:3699-3709.

21. Rotoli B, Robledo R, Luzzatto L. Decreased number of circulating BFU-Es in paroxysmal nocturnal hemoglobinuria. Blood. 1982;60: $157-159$.

22. Maciejewski JP, Sloand EM, Sato T, Anderson S, Young NS. Impaired hematopoiesis in paroxysmal nocturnal hemoglobinuria/aplastic anemia is not associated with a selective proliferative defect in the glycosylphosphatidy linositol-anchored protein-deficient clone. Blood. 1997;89:1173-1181.

23. Mukhina GL, Buckley JT, Barber JP, Jones RJ, Brodsky RA. Multilineage glycosylphosphatidylinositol anchor-deficient haematopoiesis in untreated aplastic anaemia. Br J Haematol. 2001;115: 476-482.

24. Sloand E, Kim S, Maciejewski JP, Tisdale J, Follmann D, Young NS. Intracellular interferon- $\boldsymbol{\gamma}$ in circulating and marrow $\mathrm{T}$ cells detected by flow cytometry and the response to immunosuppressive therapy in patients with aplastic anemia. Blood. 2002;100:1185-1191.

25. Bessler M, Mason P, Hillmen P, Luzzatto L. Somatic mutations and cellular selection in paroxysmal nocturnal haemoglobinuria. Lancet. 1994;343:951-953.

26. Endo M, Ware RE, Vreeke TM, et al. Molecular basis of the heterogeneity of expression of glycosyl phosphatidylinositol anchored proteins in paroxysmal nocturnal hemoglobinuria. Blood. 1996;87:2546-2557.

27. Nishimura J, Inoue $\mathrm{N}$, Wada $\mathrm{H}$, et al. A patient with paroxysmal nocturnal hemoglobinuria bearing four independent PIG-A mutant clones. Blood. 1997;89:3470-3476.

28. Mortazavi Y, Merk B, McIntosh J, et al. The spectrum of PIG-A gene mutations in aplastic anemia/paroxysmal nocturnal hemoglobinuria (AA/PNH): a high incidence of multiple mutations and evidence of a mutational hot spot. Blood. 2003;101:2833-2841.

29. Traulsen A, Pacheco JM, Dingli D. On the origin of multiple mutant clones in paroxysmal nocturnal hemoglobinuria. Stem Cells. 2007;25:3081-3084.

30. Araten DJ, Nafa K, Pakdeesuwan K, Luzzatto L. Clonal populations of hematopoietic cells with paroxysmal nocturnal hemoglobinuria genotype and phenotype are present in normal individuals. Proc Natl Acad Sci U S A. 1999;96:5209-5214.

31. Hanaoka N, Kawaguchi T, Horikawa K, Nagakura S, Mitsuta H, Nakakuma H. Immunoselection by natural killer cells of PIGA mutant cells missing stress-inducible ULBP. Blood. 2006;107:1184-1191.

32. Inoue N, Murakami Y, Kinoshita T. Molecular genetics of paroxysmal nocturnal hemoglobinuria. Int J Hematol. 2003;77:107-112.

33. Brodsky RA, Vala MS, Barber JP, Medof ME, Jones RJ. Resistance to apoptosis caused by PIG-A gene mutations in paroxysmal nocturnal hemoglobinuria. Proc Natl Acad Sci U SA. 1997;94:8756-8760.

34. Savage WJ, Barber JP, Mukhina GL, et al. Glycosylphosphatidylinositolanchored protein deficiency confers resistance to apoptosis in PNH. Exp Hematol. 2009;37:42-51.

35. Ware RE, Nishimura J, Moody MA, Smith C, Rosse WF, Howard TA. The PIG-A mutation and absence of glycosylphosphatidylinositollinked proteins do not confer resistance to apoptosis in paroxysmal nocturnal hemoglobinuria. Blood. 1998;92:2541-2550.
36. Jasinski M, Keller P, Fujiwara Y, Orkin SH, Bessler M. GATA1-Cre mediates Piga gene inactivation in the erythroid/megakaryocytic lineage and leads to circulating red cells with a partial deficiency in glycosyl phosphatidylinositol-linked proteins (paroxysmal nocturnal hemoglobinuria type II cells). Blood. 2001;98:2248-2255.

37. Rosti V. Murine models of paroxysmal nocturnal hemoglobinuria. Ann N Y Acad Sci. 2002;963:290-296.

38. Rother RP, Bell L, Hillmen, Gladwin MT. The clinical sequelae of intravascular hemolysis and extracellular plasma hemoglobin: a novel mechanism of human disease. JAMA. 2005;293:1653-1662.

39. Hill A, Rother RP, Wang X, et al. Eculizumab reduces pulmonary hypertension through inhibition of hemolysis-associated nitric oxide consumption in patients with paroxysmal nocturnal hemoglobinuria. Blood. 2008;112:486.

40. Hall C, Richards S, Hillmen P. Primary prophylaxis with warfarin prevents thrombosis in paroxysmal nocturnal hemoglobinuria $(\mathrm{PNH})$. Blood. 2003;102:3587-3591.

41. Fowkes FJ, Price JF, Fowkes FG. Incidence of diagnosed deep vein thrombosis in the general population: systematic review. Eur J Vasc Endovasc Surg. 2003;25:1-5.

42. Hill A, Richards S, Hillmen P. Recent developments in the understanding and management of paroxysmal nocturnal haemoglobinuria. $\mathrm{Br} J$ Haematol. 2007;137:181-192.

43. Wiedmer T, Hall SE, Ortel TL, Kane WH, Rosse WF, Sims PJ. Complement-induced vesiculation and exposure of membrane prothrombinase sites in platelets of paroxysmal nocturnal hemoglobinuria. Blood. 1993;82:1192-1196.

44. Hugel B, Socié G, Vu T, et al. Elevated levels of circulating procoagulant microparticles in patients with paroxysmal nocturnal hemoglobinuria and aplastic anemia. Blood. 1999;93:3451-3456.

45. Ploug M, Plesner T, Rønne E, et al. The receptor for urokinase-type plasminogen activator is deficient on peripheral blood leukocytes in patients with paroxysmal nocturnal hemoglobinuria. Blood. 1992;79:1447-1455.

46. Saso R, Marsh J, Cevreska L, et al. Bone marrow transplants for paroxysmal haemoglobinuria. Br J Haematol. 1999;104:392-396.

47. Hegenbart U, Niederwieser D, Forman S, et al. Hematopoietic cell transplantation from related and unrelated donors after minimal conditioning as a curative treatment modality for severe paroxysmal nocturnal hemoglobinuria. Biol Blood Marrow. Transplant 2003;9: 689-697.

48. Matos-Fernandez NA, Abou Mourad YR, Caceres W, KharfanDabaja MA. Current status of allogeneic hematopoietic stem cell transplantation for paroxysmal nocturnal hemoglobinuria. Biol Blood Marrow Transplant. 2009;15:656-661.

49. Yonemura Y, Kawakita M, Koito A, et al. Paroxysmal nocturnal haemoglobinuria with coexisting deficiency of the ninth component of complement: lack of massive haemolytic attack. $\mathrm{Br} J$ Haematol. 1990;74:108-113.

50. Overturf GD. Indications for the immunological evaluation of patients with meningitis. Clin Infect Dis. 2003;36:189-194.

51. Rother RP, Mojcik CF, McCroskery EW. Inhibition of terminal complement: a novel therapeutic approach for the treatment of systemic lupus erythematosus. Lupus. 2004;13:328-334.

52. Rother RP, Rollins SA, Mojcik CF, Brodsky RA, Bell L. Discovery and development of the complement inhibitor eculizumab for the treatment of paroxysmal nocturnal hemoglobinuria. Nat Biotechnol. 2007;25:1256-1264.

53. Hill A, Hillmen P, Richards SJ, et al. Sustained response and long-term safety of eculizumab in paroxysmal nocturnal hemoglobinuria. Blood. 2005;106:2559-2565.

54. Hillmen P, Muus P, Dührsen U, et al. Effect of the complement inhibitor eculizumab on thromboembolism in patients with paroxysmal nocturnal hemoglobinuria. Blood. 2007;110:4123-4128.

55. Wiedermann CJ, Stockner I. Warfarin-induced bleeding complications clinical presentation and therapeutic options. Thromb Res. 2008;122: S13-S18. 
56. Nishimura J, Kanakura Y, Ware RE, et al. Clinical course and flow cytometric analysis of paroxysmal nocturnal hemoglobinuria in the United States and Japan. Medicine (Baltimore). 2004;83:193-207.

57. Clark D, Butler SA, Braren V, Hartmann RC, Jenkins DE Jr. The kidneys in paroxysmal nocturnal hemoglobinuria. Blood. 1981;57: 83-89.

58. Tanaka YO, Anno I, Itai Y, Abe T. Paroxysmal nocturnal hemoglobinuria: MR findings. J Comput Assist Tomogr. 1993;17:749-753.

59. Rimola J, Martin J, Puig J, Darnell A, Massuet A. The kidney in paroxysmal nocturnal haemoglobinuria: MRI findings. $\mathrm{Br} J$ Radiol. 2004;77:953-956.

60. Mulopulos GP, Turner DA, Schwartz MM, Murakami ME, Clark JW. MRI of the kidneys in paroxysmal nocturnal hemoglobinuria. AJR Am J Roentgenol. 1986;146:51-52.

61. Hillmen P, Elebute O, Kelly R, et al. High incidence of progression to chronic renal insufficiency in patients with paroxysmal nocturnal hemoglobinuria. Blood. 2007;110:3678.

62. Kelly RJ, Arnold L, Richards S, et al. Successful management of intravascular breakthrough hemolysis on standard dose eculizumab therapy in PNH. Blood. 2008;112:3441.
63. Risitano AM, Notaro R, Marando L, et al. Complement fraction 3 binding on erythrocytes as additional mechanism of disease in paroxysmal nocturnal hemoglobinuria patients treated by eculizmab. Blood. 2009;113:4094-4100.

64. Fieni S, Bonfanti L, Gramellini D, Benassi L, Delsignore R. Clinical management of paroxysmal nocturnal hemoglobinuria in pregnancy: a case report and updated review. Obstet Gynecol Surv. 2006;61:593-601.

65. Ray JG, Burows RF, Ginsberg JS, Burrows EA. Paroxysmal nocturnal hemoglobinuria and the risk of venous thrombosis: review and recommendations for management of the pregnant and nonpregnant patient. Haemostasis. 2000;30:103-117.

66. Tichelli A, Socie G, Marsh J, et al. Outcome of pregnancy and disease course among women with aplastic anemia treated with immunosuppression. Ann Intern Med. 2002;137:164-172.

67. Danilov AV, Smith H, Craigo S, Feeney DM, Relias V, Miller KB. Paroxysmal nocturnal hemoglobinuria $(\mathrm{PNH})$ and pregnancy in the era of eculizumab. Leuk Res. 2009;33:4-5.

68. Kelly RJ, Arnold L, Richards SJ, et al. Successful pregnancy outcomes in paroxysmal nocturnal hemoglobinuria with long-term eculizumab treatment. Blood. 2008;112:4576.
Therapeutics and Clinical Risk Management

\section{Publish your work in this journal}

Therapeutics and Clinical Risk Management is an international, peerreviewed journal of clinical therapeutics and risk management, focusing on concise rapid reporting of clinical studies in all therapeutic areas, outcomes, safety, and programs for the effective, safe, and sustained use of medicines. This journal is indexed on PubMed Central, CAS,

\section{Dovepress}

EMBase, Scopus and the Elsevier Bibliographic databases. The manuscript management system is completely online and includes a very quick and fair peer-review system, which is all easy to use. Visit http://www.dovepress.com/testimonials.php to read real quotes from published authors.

Submit your manuscript here: http://www.dovepress.com/therapeutics-and-clinical-risk-management-journal 